\title{
Standardization of dot-enzyme-linked immmunosorbent assay for the diagnosis of bovine visceral schistosomiasis
}

\author{
Kommu Sudhakar ${ }^{1}$, G. S. Sreenivasa Murthy ${ }^{1}$ and Gaddam Rajeshwari²
}

1. Department of Veterinary Parasitology, College of Veterinary Science, P V Narsimha Rao Telangana Veterinary University, Rajendranagar, Hyderabad, Telangana, India; 2. Department of Veterinary Parasitology, Teaching Veterinary Clinical Complex, College of Veterinary Science, P V Narsimha Rao Telangana Veterinary University, Rajendranagar, Hyderabad, Telangana, India.

Corresponding author: Kommu Sudhakar, e-mail: vety0756@gmail.com, Co-authors: GSSM: gssmurthy26@rediffmail.com, GR: rajeshwari.g04@gmail.com Received: 29-11-2016, Accepted: 30-04-2017, Published online: 21-05-2017

doi: 10.14202/vetworld.2017.536-541 How to cite this article: Sudhakar K, Murthy GSS, Rajeshwari G (2017) Standardization of dot-enzyme-linked immmunosorbent assay for the diagnosis of bovine visceral schistosomiasis, Veterinary World, 10(5): 536-541.

\begin{abstract}
Aim: Bovine visceral schistosomiasis has been reported as an important disease entity as it affects animal health, productivity, causes economic losses due to liver condemnation, and produces a high morbidity. This study was conducted to standardize an easy, reliable dot-enzyme-linked immmunosorbent assay (ELISA) for the diagnosis of visceral schistosomiasis caused by Schistosoma spindale and to know the prevalence rate in and around Hyderabad.
\end{abstract}

Materials and Methods: A dot-ELISA was standardized in the laboratory using whole worm antigen (WWA) and excretorysecretory antigen (ESA) of $S$. spindale. The standardized test was used for the diagnosis of bovine visceral schistosomiasis at field level. The sensitivity and specificity of the test was compared with counter current immunoelectrophoresis. In total, 288 sera (125 cattle and 163 buffalo) were screened by dot-ELISA.

Results: The dot-ELISA detected $32.63 \%$ of infection (94/288) using WWA and $40.62 \%$ of infection (117/288) using ESA. In cattle, the prevalence rate was 32.80\% (41/125) using WWA and 40.80\% (51/125) of infection. Similarly, in buffaloes, the prevalence rate was 32.51\% (53/163) using WWA and 40.49\% (66/163) of infection using ESA. The overall sensitivity of dot-ELISA was $76.74 \%$ and $80.48 \%$ with WWA and ESA, respectively, and specificity was $73.3 \%$ and $78.57 \%$ in WWA and ESA, respectively.

Conclusion: As ante-mortem diagnosis of visceral schistosomiasis is difficult in subclinical conditions, dot-ELISA can be used as a reliable immunodiagnostic test for diagnosis at field level.

Keywords: dot-enzyme-linked immmunosorbent assay, excretory-secretory antigen, Schistosoma spindale, whole worm antigen.

\section{Introduction}

Schistosomiasis has been recognized as one of the major parasitic diseases of livestock and human beings. Bovine visceral schistosomiasis in Indian subcontinent is primarily caused by two Schistosoma species such as Schistosoma spindale and Schistosoma indicum. High rates of prevalence of subclinical infections cause significant losses due to long-term effects on animal growth, productivity, reduced conception and pregnancy rates [1], and increased susceptibility to other parasitic or bacterial diseases $[1,2]$. Schistosomiasis also causes loss due to liver condemnation in animal species [3]. All fecal examination methods for diagnosis are found to be less sensitive [4]. S. spindale infection is generally underdiagnosed, as the eggs in fecal samples of

Copyright: Sudhakar, et al. Open Access. This article is distributed under the terms of the Creative Commons Attribution 4.0 International License (http://creativecommons.org/licenses/ by/4.0/), which permits unrestricted use, distribution, and reproduction in any medium, provided you give appropriate credit to the original author(s) and the source, provide a link to the Creative Commons license, and indicate if changes were made. The Creative Commons Public Domain Dedication waiver (http:// creativecommons.org/publicdomain/zero/1.0/) applies to the data made available in this article, unless otherwise stated. affected animals are usually masked by high mucus content and eggs in feces also hatch immediately on contact with water. Previous studies on the incidence and prevalence of schistosomiasis in India were based solely on nasal scrapings, fecal examination, and nasal and mesenteric cuttings in cattle [5]. A coprological survey in water buffaloes of Kurigram district of Bangladesh indicated $1.27 \%$ S. indicum and $0.85 \%$ $S$. spindale infection [6]. This trematode was more likely to co-occur with other gastrointestinal parasites (i.e., Dicrocoelium spp., Paramphistomum spp., Strongyle spp., Eimeria spp., and Entamoeba spp.), and statistical analysis revealed that female cattle are less likely to get $S$. spindale infection as compared to male cattle, and cattle weighing lower than $200 \mathrm{~kg}$ were significantly at higher risk than those higher than $200 \mathrm{~kg}$ in Malaysia [7].

The antigens from schistosome eggs, cercariae, and schistosomula have been isolated and found useful for various purposes. The secretory-excretory products, tegument, and gut proteins of adult schistosomes are the source of potential immunogens. Very less attempts were made toward the identification, characterization, and use of antigens of $S$. spindale 
toward these objectives. The immunodiagnosis can be considered as an essential mean for the confirmatory diagnosis.

Hence, the present study was conducted to standardize a serological test (dot-enzyme-linked immmunosorbent assay [ELISA]) for the diagnosis of visceral schistosomiasis and to evaluate it for the diagnosis of the disease in field levels.

\section{Materials and Methods}

\section{Ethical approval}

Two rabbits were used for raising hyperimmune sera (HIS) against whole worm antigen (WWA) and ES antigen (ESA) of $S$. spindale (permission was accorded by Ethical Committee bearing Ref: 698/ CPCSEA dated October 01, 2002 F.No. 25/60/2010AWD/Veterinary College/Hyderabad).

\section{Collection of adult schistosomes}

The mesenteric veins of the freshly collected bovine intestines from slaughter house were examined against sunlight, and the adult schistosomes were collected carefully in sterile phosphate-buffered saline (PBS). After three washings in sterile PBS, the parasites were segregated species wise based on their morphological characters under light microscopy. S. spindale male worms were identified by their smooth cuticle with 3-5 number of testes and female worms lodged in gynecophoric canal of male worms containing spindle-shaped eggs in the uterus with terminal spine at one end. $S$. indicum worms can be separated based on rough cuticle (tuberculated/spiny) with 5-7 testes and the female worms containing ovalshaped eggs with terminal spine at one end. After species separation, $S$. spindale worms were used for further studies.

Around 500 freshly collected live flukes were washed thoroughly in normal saline and transferred to $2 \mathrm{ml}$ of $0.01 \mathrm{M}$ PBS (pH 7.4) containing ampicillin $(40 \mathrm{mg})$, amikacin $(40 \mathrm{mg})$, sodium azide $(0.1 \%)$, protease inhibitor, and phenylmethylsulfonyl fluoride (PMSF $1 \mathrm{mM}$ ). The worms were incubated at $37^{\circ} \mathrm{C}$ in an incubator with 5\% carbon dioxide for overnight. On next day morning, the incubated fluid containing excretory-secretory (ES) products was collected and centrifuged at $13,000 \mathrm{rpm}$ at $4^{\circ} \mathrm{C}$ for $30 \mathrm{~min}$. The supernatant was collected and stored at $-20^{\circ} \mathrm{C}$.

Similarly, the WWA were prepared by taking around 500 worms of $S$. spindale after washing thrice in PBS (pH 7.4) and triturated with $5 \mathrm{ml}$ of PBS (pH 7.4) using glass tissue homogenizer (Potter Elvehjem glass Teflon) for $10 \mathrm{~min}$ at $4^{\circ} \mathrm{C}$. The contents were sonicated at $20 \mathrm{kHz}$ for 10 cycles for $90 \mathrm{~s}$ each with an interval of $1 \mathrm{~min}$ in between. The contents were centrifuged at $9500 \times g$ for $15 \mathrm{~min}$ at $4{ }^{\circ} \mathrm{C}$ in a high-speed centrifuge (C30 Remi, India). The supernatant was collected and preserved by adding one drop of $1 \%$ sodium azide and $1 \mathrm{mM}$ of PMSF at $-20^{\circ} \mathrm{C}$, after making the solution into several aliquots.
The protein concentration of antigens was estimated as per the method of Lowry et al. (1951) using protein estimation kit obtained from Genei, Bengaluru.

\section{Protocol for raising of hyper immune sera}

WWA $(0.25 \mathrm{ml})$ containing $3.88 \mathrm{mg} / \mathrm{ml}$ of protein along with equal volume of Freund's complete adjuvant (FCA) was injected subcutaneously into one rabbit. Similarly, $0.3 \mathrm{ml}$ of ESA $(2.01 \mathrm{mg} / \mathrm{ml}$ of protein) along with $0.3 \mathrm{ml}$ of FCA was injected subcutaneously to another rabbit. Four booster doses containing either WWA $(0.25 \mathrm{ml})$ or ESA $(0.3 \mathrm{ml})$ along with equal volume of Freund's incomplete adjuvant were administered subcutaneously to the respective rabbits at weekly intervals. The animals were bled by the puncture of marginal ear vein and checked for antibody $(\mathrm{Ab})$ response by agar gel precipitation test before administering each booster injection. Blood was collected by cardiac puncture on the $10^{\text {th }}$ day of last booster injection. Serum was separated under sterile conditions, and complement inactivation was done by keeping in water bath at $56^{\circ} \mathrm{C}$ for $30 \mathrm{~min}$. Sera were tested for the presence of antibodies using corresponding antigen with double immunodiffusion (DID) and counter current immunoelectrophoresis (CCIEP) techniques. The sera containing antibodies for respective antigens were made into aliquots and stored at $-20^{\circ} \mathrm{C}$ till further use.

The immunoprecipitation reactions between antigen (WWA/ESA) with respective hyperimmune sera developed in rabbits were detected by DID and CCIEP.

\section{Standardization of dot-ELISA using HIS}

Dot-ELISA was standardized by charging $1.5 \mu 1$ of different dilutions of the antigens $(3.88,1.94,0.97$, and $0.485 \mathrm{mg} / \mathrm{ml}$ of WWA and 2.01, 1, 0.5, and $0.25 \mathrm{mg} / \mathrm{ml}$ of ESA) on to nitrocellulose (NC) membrane of $1 \mathrm{~cm}^{2}$, which was previously washed in autoclaved double-distilled water and dried at room temperature. The strips were kept in the incubator at $37^{\circ} \mathrm{C}$ for $1 \mathrm{~h}$. The serum samples (HIS and negative controls) at different dilutions (1:50, 1:100, 1:200, and $1: 400$ ) with $0.05 \%$ Tween 20 in PBS (PBST) were allowed to react with the antigen-loaded strip for $1 \mathrm{~h}$ at $37^{\circ} \mathrm{C}$ followed by washing of NC strips for 6 times in PBST (pH 7.4). The NC membranes were treated with goat anti-rabbit immunoglobulin $\mathrm{G}$ (IgG) horseradish peroxidase (HRP) conjugate at different dilutions $(1: 1000,1: 1500,1: 2000,1: 2500$, and 1:5000) with PBST followed by washing 4 times each for 10 min. Subsequently, the $\mathrm{Ab}$ and conjugate-treated antigen-coated $\mathrm{NC}$ membrane strips were finally treated with the chromogen substrate di-aminobenzidine (DAB - $0.5 \mathrm{mg} / \mathrm{ml}$, cobalt chloride $-0.2 \mathrm{mg} / \mathrm{ml}$, Tris buffer $-0.05 \mathrm{M}$, sodium chloride $-0.15 \mathrm{M}$, and $3 \%$ hydrogen peroxide $-15 \mu 1$ ) in dark for 2-3 min. The dark brown spot appeared on the NC strip indicated positive reaction. The reaction was stopped with double-distilled water. Irrespective of the intensity 
of the color, the reaction was considered as positive and was documented by digital zoom camera (Nikon COOL PIX AW 120).

The lowest possible concentration of either WWA or ESA giving positive reaction with highest possible dilution of respective HIS and goat anti-IgG $\mathrm{HRP}$ conjugate in the form of brown spot on $\mathrm{NC}$ membrane, irrespective of color intensity, was considered as cutoff value and the same was taken as standard dilution for the screening of bovine serum samples from the field.

\section{CCIEP}

CCIEP was also done to compare the sensitivity and specificity of the standardized dot-ELISA. Agarose was prepared in Tris borate buffer ( $\mathrm{pH}$ 8.2). $1 \mathrm{~g}$ of agarose special, low EEO (HIMEDIA), was boiled in $100 \mathrm{ml}$ of Tris borate buffer. $5 \mathrm{ml}$ of molten gel was poured on to glass slides and allowed to solidify. Two wells of $4 \mathrm{~mm}$ diameter were cut with a well distance of $5 \mathrm{~mm}$ for trials with corresponding hyperimmune sera. The slides were connected by a filter paper wick dipped in buffer. A current of $50 \mathrm{~mA}$ per slide was applied and results were recorded after 90 min.

The sensitivity and specificity of dot-ELISA was measured by standardized dot-ELISA procedure described as earlier.

To evaluate the sensitivity and specificity of CCIEP, two rows of wells, each row having seven wells (each well having $4 \mathrm{~mm}$ diameter and a distance of $5 \mathrm{~mm}$ in between), were punched on agar slide. Wells were cut and the bases were sealed with molten agar. The well to be located on the cathode side was filled with antigens of $S$. spindale and other (anodic well) with corresponding hyperimmune serum for evaluation of the test and later with sera from known positive or negative animal. Electrophoresis was carried out in suitable trough containing electrophoresis buffer. Both ends of the slides were connected with a filter paper wick dipped in buffer. A current of $50 \mathrm{~mA}$ per slide was applied for $90 \mathrm{~min}$. The slides were washed in normal saline and stained with Coomassie brilliant blue.

The sensitivity of dot-ELISA and CCIEP was measured by screening 33 known positive sera collected from cattle which were later confirmed by finding schistosomes in the mesentery on post-slaughter examination.

The specificity of laboratory dot-ELISA and CCIEP was measured by screening the sera of cattle reared in confined area and was found negative for the clinical symptoms of schistosomiasis or any other helminth ova in the feces.

The laboratory-standardized dot-ELISA was tested for its efficacy in the diagnosis of bovine visceral schistosomiasis and further evaluation of dotELISA at field level.

Around 288 bovine sera were collected from different parts in and around Hyderabad, Telangana state (erstwhile Andhra Pradesh) and subjected to standardized dot-ELISA with different antigens.

\section{Results}

The dot-ELISA was standardized at an antigen concentration of $91 \mathrm{ng}$ for WWA and $93 \mathrm{ng}$ for ESA which gave brown color dot with the chromogen substrate DAB after reacting with the serum and conjugate at 1:100 and 1:2000 dilutions, respectively (Figure-1).

The overall sensitivity of dot-ELISA was determined as $76.74 \%$ and $80.48 \%$ with WWA and ESA, respectively, and specificity was $73.33 \%$ and $78.57 \%$ in WWA and ESA, respectively (Tables-1 and 2). A total of 288 bovine sera (125 cattle and 163 buffalo) were examined using the standardized dot-ELISA test. Out of the 125 sera samples screened in cattle, 41 were positive indicating $32.80 \%$ infection with WWA and 51 were positive indicating $40.80 \%$ infection with ESA. Whereas in the screening of 163 sera samples in buffalo, 53 were positive indicating $32.51 \%$ infection with WWA and 66 were positive indicating $40.49 \%$ infection with ESA. The overall percentage infection of schistosomiasis in bovines was recorded as $32.63 \%$ (94/288) using WWA and 40.62\% (117/288) using ESA (Table-3).

\section{Discussion}

Schistosomiasis has been recognized as one of the major parasitic diseases of livestock and human beings. S. spindale is the major cause of visceral schistosomiasis among bovines [8]. Routine diagnostic methods have poor sensitivity and thus underestimate the actual prevalence which ultimately interferes with the control strategies [9]. The prevalence of schistosomiasis was very high based on mesenteric worm technique when compared to the existing reports based on fecal egg detection techniques [9]. This study emphasizes the need for developing alternate fieldlevel diagnostic tests for schistosomiasis because the

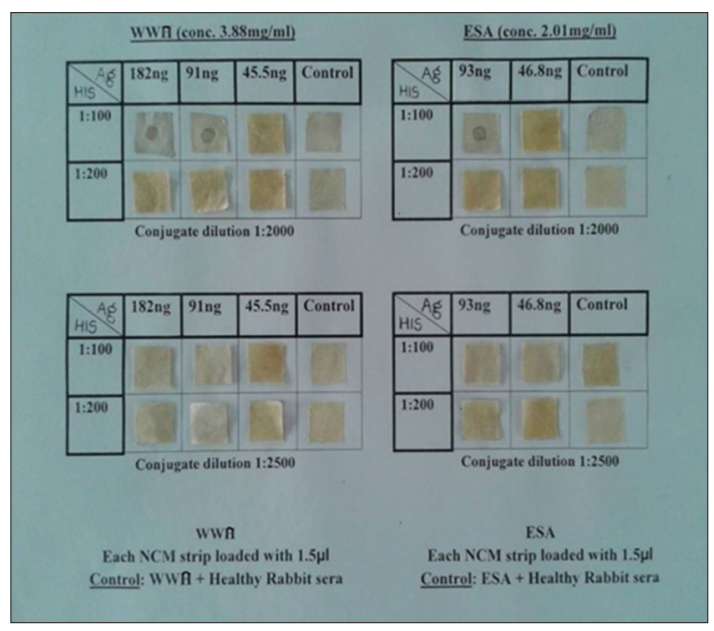

Figure-1: Optimum concentration of antigen (whole worm antigen/excretory-secretory antigen), hyperimmune sera, and conjugate (goat anti-rabbit immunoglobulin G horseradish peroxidase) showing a brown dot. 
routine method of diagnosis generally underdiagnoses many infections at field level.

Dot-ELISA, a modification of the standard ELISA assay, offers a practical tool for field studies. Moreover, the control of a disease is possible only based on the sensitivity and specificity of the diagnostic method. Dot-ELISA technique was first applied for detecting the anti-leishmania antibodies in human beings.

Different immunological techniques, namely, latex agglutination test, sandwich ELISA, and dot-ELISA were evaluated for the diagnosis of Schistosoma haematobium infection in Egypt using purified soluble egg antigen, and the tests were proved to be valuable and can be used as an easy, fast, and accurate diagnostic technique [10].

In the present investigation, $91 \mathrm{ng}$ of WWA and $93 \mathrm{ng}$ of ESA of $S$. spindale dotted on NCM could give a positive brown-colored dot with 1:100 dilution of HIS when coupled with goat anti-rabbit IgG HRP followed by reaction with DAB substrate.

Various authors have tried to develop different kinds of ELISA tests including dot-ELISA which required antigens in nanogram quantities and the present findings were similar to them $[8,11-15]$.

The dot-ELISA using WWA could detect 23 positive cases out of 33 known positive bovine sera, indicating an overall sensitivity of $76.74 \%$ whereas the same test with ESA detected 25 positive cases among
33 known positive bovine sera showing an overall sensitivity of $80.48 \%$. Similarly, dot-ELISA using WWA gave 4 positive reactions with 11 known negative sera showing $73.33 \%$ of specificity. Whereas ESA reacted positively with 3 out of 11 known bovine sera negative for schistosomiasis indicating $78.57 \%$ of specificity. Our results were in correlation with the findings of earlier workers $[8,13,15]$. In the present study, schistosomiasis was detected in $32.63 \%$ and $40.62 \%$ of bovines in Telangana, using WWA and ESA in dot-ELISA, respectively. These results are comparable with that of Lakshmanan et al. [8] as 32\% of bovines in Kerala using ESA in dot-ELISA. The results are almost in agreement with that of Lakshmanan et al. [8], but they have not categorized as cattle or buffaloes like in the present findings.

Adult worm homogenates of $S$. indicum and $S$. spindale were prepared and subjected to sodium dodecyl sulfate polyacrylamide gel electrophoresis to know the polypeptide profiles of the prepared antigen. Finally, four immunodominant proteins $(45,40,28$, and $15 \mathrm{kDa}$ ) were obtained and no significant variation was found between the immunodominant proteins $S$. indicum and $S$. spindale. As mixed infection is common in bovines, in this study, the mixed worm antigen was used for the detection of humoral immune status of infected cattle, sheep, and goats [12].

Cattle sera showed $32.8 \%(41 / 125)$ infection with WWA, 40.80\% (51/125) infection with ESA, whereas

Table-1: Sensitivity studies on dot-ELISA in S. spindale infection (WWA and ESA).

\begin{tabular}{lcccc}
\hline Antigen & $\begin{array}{c}\text { Number of } \\
\text { samples (known positive) }\end{array}$ & Dot-ELISA (positive) & $\begin{array}{c}\text { Dot-ELISA negative (false } \\
\text { negative) }\end{array}$ & $\begin{array}{c}\text { Sensitivity } \\
\text { percentage }\end{array}$ \\
\hline WWA & 33 & $23^{\mathrm{a}}$ & 10 & 76.74 \\
ESA & 33 & $25^{\mathrm{a}}$ & 8 & 80.48 \\
\hline
\end{tabular}

Sensitivity: True positive/True positive+False negative $\times 100$. The values superscripted with similar alphabets are not significantly $(p \geq 0)$ different. WWA=Whole worm antigen, ESA=Excretory-secretory antigen, ELISA=Enzyme-linked immmunosorbent assay, S. spindale=Schistosoma spindale

Table-2: Specificity studies on dot-ELISA in S. spindale infection (WWA and ESA).

\begin{tabular}{lcccc}
\hline Antigen type & $\begin{array}{c}\text { Number of } \\
\text { samples (known negative) }\end{array}$ & Dot-ELISA (negative) & $\begin{array}{c}\text { Dot-ELISA positive (false } \\
\text { positive) }\end{array}$ & $\begin{array}{c}\text { Specificity } \\
\text { percentage }\end{array}$ \\
\hline WWA & 11 & 7 & 4 & 73.33 \\
ESA & 11 & 8 & 3 & 78.57 \\
\hline
\end{tabular}

Specificity: True negatives/True negatives+False positives $\times 100$. The values superscripted with similar alphabets are not significantly $(p \geq 0)$ different. WWA =Whole worm antigen, ESA=Excretory-secretory antigen, ELISA=Enzyme-linked immmunosorbent assay, S. spindale=Schistosoma spindale

Table-3: Detection of S. spindale infection by dot-ELISA using WWA and ESA.

\begin{tabular}{|c|c|c|c|c|c|c|c|c|c|}
\hline \multirow[t]{2}{*}{ Species } & \multirow{2}{*}{$\begin{array}{l}\text { Number } \\
\text { of sera } \\
\text { screened }\end{array}$} & \multicolumn{4}{|c|}{ Dot-ELISA using WWA } & \multicolumn{4}{|c|}{ Dot-ELISA using ESA } \\
\hline & & Positive & Negative & $\begin{array}{l}\text { Percent- } \\
\text { positive } \\
\text { infection }\end{array}$ & $\begin{array}{l}\text { Percent- } \\
\text { negative } \\
\text { infection }\end{array}$ & Positive & Negative & $\begin{array}{l}\text { Percent- } \\
\text { positive } \\
\text { infection }\end{array}$ & $\begin{array}{c}\text { Percent-negative } \\
\text { infection }\end{array}$ \\
\hline Cattle & 125 & $41^{\text {by }}$ & 84 & 32.80 & 62.20 & $51^{\text {ay }}$ & 74 & 40.80 & 59.20 \\
\hline Buffalo & 163 & $53^{b x}$ & 110 & 32.51 & 67.49 & $66^{\mathrm{ax}}$ & 97 & 40.49 & 59.51 \\
\hline Total & 288 & 94 & 194 & 32.63 & 67.37 & 117 & 171 & 40.62 & 59.38 \\
\hline
\end{tabular}

${ }^{a b}$ The positive values bearing different superscriptions between the species differ significantly ( $\left.p \leq 0.05\right)$. ${ }^{x}$ The positive values bearing different superscriptions between the tests differ significantly $(p \leq 0.05)$. WWA $=$ Whole worm antigen, $\mathrm{ESA}=$ Excretory-secretory antigen, ELISA=Enzyme-linked immmunosorbent assay, S. spindale=Schistosoma spindale 
buffalo sera showed $32.51 \%(53 / 163)$ infection with WWA and 40.49\% (66/163) infection with ESA. Use of WWA in dot-ELISA detected anti-schistosomal antibodies in cattle indicating $90.93 \%$ (291/320) [11]. The results of the present findings in cattle are not corroborated with that of Sumanth et al. [11] who advocated positivity in latter cases either due to light infection or their previous exposure to infection, most probably this was due to non-specificity of the reaction.

In this study, buffaloes showed $32.51 \%$ and $40.49 \%$ infection using WWA and ESA, respectively. Results cannot be compared, due to the paucity of literature, with various authors as they reported either in cattle or in general bovines, but the present study shows a significant infection rate of schistosomiasis in cattle and buffaloes separately in Telangana state.

As a whole, the dot-ELISA with ESA was found to be more sensitive (80.48\%) and specific (78.57\%) than WWA in detecting antibodies to $S$. spindale infection in bovines. The superiority of ESA in serodiagnosis has been observed by several workers for detecting other trematode infections, namely, Clonorchis sinensis, Paragonimus spp., Fasciola hepatica [16], Opisthorchis felineus [17], and Paramphistomum cervi [18]. This ESA could be useful for rapid screening of a large number of field samples, with wide applicability.

Even though some differences have been identified regarding the sensitivity and specificity of the dot-ELISA in its different applications $[19,20]$, the test was proved to be highly stable, does not require specialized tools to analyze the results, cost-effective, and can be simultaneously applied on large-scale screening of samples according to Chieffi et al. [21].

\section{Conclusion}

It is concluded that $S$. spindale antigens were useful for serodiagnosis of visceral schistosomiasis in bovines by dot-ELISA. Dot-ELISA using ESA could also be considered for ante-mortem diagnosis of schistosomal infection with a high degree of reliability under field conditions. This technique could form the basis for evolving a uniform system of seromonitoring of $S$. spindale in local bovine population, in turn enabling implementation of a suitable disease control strategy in the country.

\section{Authors' Contributions}

GSSM and KS have designed the concept and supervised the plan of work and also have prepared the manuscript. GR helped in collecting intestines from slaughter house and provided technical, as well as material support. GSSM analyzed and interpreted the data. All authors read and approved the final manuscript.

\section{Acknowledgments}

The authors would like to thank the personnel of Chengicherla main slaughterhouse for their kind assistance and authors would like to thank $\mathrm{P} V$ Narsimha Rao Telangana Veterinary University, formerly Sri Venkateshwara Veterinary University, for providing necessary facilities to conduct this research work.

\section{Competing Interests}

The authors declare that they have no competing interests.

\section{References}

1. McCauley, E.H., Majid, A.A. and Tayeb, A. (1984) Economic evaluation of the production impact of bovine schistosomiasis and vaccination in the Sudan. Prev. Vet. Med., 2(6): 735-754.

2. Dargie, J.D. (1980) The pathogenesis of S. Bovis infection in Sudanese cattle. Trans. R. Soc. Trop. Med. Hyg., 74: 560-562.

3. Kadam, S.S., Sardeshpande, P.D. and Ajinkya, S.M. (1977) Hepatic schistosomiasis in sheep and goats. Bombay Vet. Col. Mag., 22: 14-15.

4. Agrawal, M.C., Vohra, S., Gupta, S. and Singh, K.P. (2004) Prevalence of helminthic infections in domestic animals in Madhya Pradesh. J. Vet. Parasitol., 18: 147-149.

5. Sumanth, S., D'Souza, P.E. and Jagannath, M.S. (2004) A study of nasal and visceral schistosomosis in cattle slaughtered at an abattoir in Bangalore, South India. Rev. Sci. Tech. Int. Off. Epizoot., 23(3): 937-942.

6. Mamun, M.A.A., Begum, N. and Mondal, M.M.H. (2011) A coprological survey of gastro-intestinal parasites of water buffaloes (Bubalus bubalis) in Kurigram district of Bangladesh. J. Bangladesh Agrc. Univ., 9(1): 103-110.

7. Tan, T.K., Low, V.L., Lee, S.C., Panchadcharam, C., Kho, K.L., Koh, F.X. and Lim, Y.A.L. (2015) Detection of Schistosoma spindale ova and associated risk factors among Malaysian cattle through coprological survey. Jpn. J. Vet. Res., 63(2): 63-71.

8. Lakshmanan, B., Devada, K., Joseph, S., Binu, M.B. and Kuttan, K. (2016) Efficacy of dot-ELISA using different antigens in detecting anti-schistosome antibodies among bovines in field conditions. J. Parasit. Dis., 40(1): 189-193.

9. Lakshmanan, B., Rauoof, A., Fawaz, M. and Subramanian, H. (2011) Abattoir survey of Schistosoma spindale infection in Thrissur. J. Vet. Anim. Sci., 42: 53-54.

10. Mahfouz, A., Mahana, N., Rabee, I. and El-Amir, A. (2011) Evaluation of different immunological techniques for diagnosis of schistosomiasis Haematobium in Egypt. Life Sci. J., 8(4): 858-867.

11. Sumanth, S., D'Souza, P.E. and Jagannath, M.S. (2003) Immunodiagnosis of nasal and visceral schistosomosis in cattle by dot-ELISA. Indian Vet. J., 80(6): 495-498.

12. Singh, A. and Chaudhri, S.S. (2004) Visceral schistosomiasis of domestic animals in India: Humoral immune status of infected cattle, sheep and goats against major polypeptide antigens of Schistosoma indicum and S. spindale. Parasite Immunol., 26(4): 167-175.

13. Vohra, S., Agrawal, M.C. and Malik, Y.P.S. (2006) DotELISA for diagnosis of caprine schistosomosis. Indian J. Anim. Sci., 76(12): 988-991.

14. Divya, S.P.I., Bindu, L., Abhinay, G., Joseph, S. and Subramanian, H. (2011) Protein Profiling of Schistosoma spindale Using SDS-PAGE. Parasitology for End Users Compendium-Cum-Souvenir XXI National Congress of Veterinary Parasitology. p32-33.

15. Murthy, G.S., D'Souza, P.E. and Isloor, K.S. (2013) Evaluation of a polyclonal antibody based sandwich ELISA for the detection of faecal antigens in Schistosoma spindale infection in bovines. J. Parasitic Dis., 37(1): 47-51.

16. Awad, W.S., Ibrahim, A.K. and Salib, F.A. (2009) Using indirect ELISA to assess different antigens for the 
serodiagnosis of Fasciola gigantica infection in cattle, sheep and donkeys. Res. Vet. Sci., 86(3): 466-471.

17. Gómez-Morales, M.A., Ludovisi, A., Amati, M. and Pozio, E. (2013) Validation of an excretory/secretory antigen based-ELISA for the diagnosis of Opisthorchis felineus infection in humans from low trematode endemic areas. Plos One., 8: 2267.

18. Anuracpreeda, P., Poljaroen, J., Chotwiwatthanakun, C., Tinikul, Y. and Sobhon, P. (2013) Antigenic components, isolation and partial characterization of excretion-secretion fraction of Paramphistomum cervi. Exp. Parasitol., 133(3): 327-333.
19. Shukla, N., Husain, N., Agarwal, G.G. and Husain, M. (2008) Utility of Cysticercus fasciolaris antigen in dot ELISA for the diagnosis of neurocysticercosis. Indian $J$. Med. Sci., 62(6): 222.

20. Blanco, R.M., Takei, K. and Romero, E.C. (2009) Leptospiral glycolipoprotein as a candidate antigen for serodiagnosis of human leptospirosis. Lett. Appl. Microbiol., 49(2): 267-273.

21. Chieffi, P.P., Santos, S.V.D., Queiroz, M.L.D. and Lescano, S.A.Z. (2009) Human toxocariasis: Contribution by Brazilian researchers. Rev. Inst. Med. Trop. São. Paulo., 51(6): 301.

$* * * * * * * *$ 